\title{
Astrocyte stress precedes demyelination in cerebral X-ALD
}

Cerebral X-linked adrenoleukodystrophy (X-ALD) is a genetic disorder characterized by accumulation of very long chain fatty acids, which ultimately leads to demyelination. The mechanisms that trigger demyelination in X-ALD are unclear, but a new study published in Neuropathology and Applied Neurobiology suggests that astrocyte stress, signified by the expression of heat shock proteins (HSPs), is involved in this process.

"HSPB5 expressed by oligodendrocytes seems to have an important role in the initiation of inflammation and demyelination in multiple sclerosis (MS), and unpublished findings from our group revealed the presence of HSPB5-positive glial cells in brain samples from patients with X-ALD," explains Anna Lena Görtz, who shares first authorship of the paper with Laura Peferoen. "These findings prompted us to gain more insight into the involvement of HSPs in the pathogenesis of X-ALD, using MS as a comparison."

The researchers performed immunohistochemistry on sections of brain tissue obtained at autopsy from five individuals with cerebral X-ALD. Expression of four small HSPs (sHSPs), HSPB1, HSPB5, HSPB6 and HSPB8, was found to be elevated in preactive X-ALD lesions — that is, brain areas containing hypertrophic astrocytes and small numbers of activated microglia and macrophages, but no signs of demyelination. The upregulated sHSPs were localized to cells expressing the astrocytic marker glial fibrillary acidic protein.

"Up to now, the primary focus in cerebral X-ALD has been on oligodendrocytes - the myelin-forming cells - and the reaction of astrocytes was interpreted within the frame of the inflammatory response," says Görtz. "However, the early involvement of stress responses in astrocytes suggests a major, previously unknown role for this cell type in the initiation of inflammatory demyelination in cerebral X-ALD."

In MS, the pro-inflammatory response that results in demyelination is thought to involve IFN- $\gamma$, but Görtz and colleagues found no evidence of IFN- $\gamma$ activation in $\mathrm{X}$-ALD lesions, indicating that the mechanisms underlying myelin loss differ between the two diseases. The researchers plan to further explore the role of astrocyte stress in cerebral $\mathrm{X}$-ALD, and to examine whether astrocytes and HSPs might be harnessed therapeutically in the early stages of the disease.

\section{Heather Wood}

ORIGINAL ARTICLE Görtz, A. L. et al. Heat shock protein expression in cerebral X-linked adrenoleukodystrophy reveals astrocyte stress prior to myelin loss. Neuropathol. Appl. Neurobiol. http://dx.doi.org/10.1111/nan.12399 (2017) 